\title{
CORRESPONDENCE
}

\section{Cough in patients after stroke}

\section{To the Editors:}

WARD et al. [1] are to be congratulated on their new and important findings concerning cough in patients after stroke. They measured a large number of ventilatory parameters during voluntary and reflex cough, and compared the values in stroke patients and healthy controls.

Although the relationship of voluntary cough after stroke with the risk of aspiration has been analysed extensively, this seem to be the first detailed study of reflex cough, induced in these experiments by tartaric acid aerosol. This is important because, should material be aspirated into the lower airways, it is reflex cough that will remove the aspirate and lessen the chance of pneumonia.

However, by definition, both voluntary and reflex cough start with an inspiration [2], which would tend to cause rather than prevent aspiration; of course, the subsequent expirations should remove any foreign material from the lungs. The reflex that prevents aspiration is the expiration reflex from the larynx; this, with initial glottal closure, has no preceding inspiration [3]. Several studies have shown that stroke patients may lose their voluntary cough but retain their expiration reflex and, if this is the case, the risk of aspiration is considerably smaller $[4,5]$.

The authors made an unexpected and important observation that, in stroke patients, the force of expiratory efforts (as assessed by gastric pressure) was considerably reduced with voluntary cough, but not with reflex cough. However, the expiratory flow parameters were reduced in both. Thus, reflex cough, as assessed by the force of expiratory efforts, is not greatly reduced after stroke, but it may be less effective. They suggest that the imbalance between expiratory pressures and flows with reflex cough may be due to ineffective coordination of different muscle groups following cerebral injury. Another possibility is that the tartaric acid aerosol is stimulating not only reflex cough from the lower airways, but also glottal constrictor reflexes from the larynx. These are well established with tartaric acid aerosol $[4,5]$ and might decrease expiratory flow rates during the expulsive phase of cough.

It is a pity that, in general, only mean values are given. It would be interesting to know if some individual patients lost voluntary cough but maintained reflex cough (although the observations mentioned above make this likely). The opposite, loss of reflex and maintenance of voluntary cough, seems unlikely on theoretical grounds, but would be an important observation. Perhaps individual results could have been made available online.

Like all good papers, this one describes significant new observations and points to future research. We are sure it will be done.

\author{
J.G. Widdicombe* and W.R. Addington ${ }^{\#}$ \\ *University of London, London, UK, and "Melbourne, FL, \\ USA.
}

Correspondence: J.G. Widdicombe, 116 Pepys Road, London SW20 8NY, UK. E-mail: JohnWiddicombeJ@aol.com

Statement of Interest: Statements of interest for both authors can be found at www.erj.ersjournals.com/site/misc/statements. xhtml

\section{REFERENCES}

1 Ward K, Seymour J, Steier J, et al. Acute ischaemic hemispheric stroke is associated with impairment of reflex in addition to voluntary cough. Eur Respir J 2010; 36: 1383-1391.

2 Morice AH, Fontana GA, Belvisi MG, et al. ERS guidelines on the assessment of cough. Eur Respir J 2007; 29: 1256-1276.

3 Widdicombe J, Fontana G. Cough: what's in a name? Eur Respir J 2006; 28: 10-15.

4 Stephens RE, Addington WR, Widdicombe JG. Effect of acute unilateral middle cerebral artery infarcts on voluntary cough and the laryngeal cough reflex. Am J Phys Med Rehab 2003; 82: 379-383.

5 Addington WR, Stephens RE, Goulding RE. Assessing the laryngeal cough reflex and the risk of developing pneumonia after stroke: an interhospital comparison. Stroke 1999; 30: 1203-1207.

DOI: $10.1183 / 09031936.00083310$

\section{From the authors:}

We thank J.G. Widdicombe and W.R. Addington for their interest in our work on cough impairment in acute hemispheric stroke patients. In our paper, we proposed that the high reflex cough pressures but impaired cough flow rates demonstrated by the stroke patients could be due to poor coordination between the upper airway, chest and abdominal muscle groups [1]. However, we acknowledge that the reflex cough flows could also be reduced by laryngeal constriction secondary to tartaric acid aerosol inhalation. The ideal way to test this hypothesis would be to image the larynx during the tests, but we have not yet developed a method for doing this.

J.G. Widdicombe and W.R Addington were also interested to know whether some patients had lost voluntary cough but maintained reflex cough, or vice versa. These data are given in figure 1. Of 18 stroke patients tested, two could produce neither voluntary cough nor reflex cough in response to $20 \%$ tartaric acid. One patient was able to produce voluntary cough but had no reflex cough response. One more patient with no reflex cough response was able to produce voluntary cough. Although, in general, there is a good relationship between 


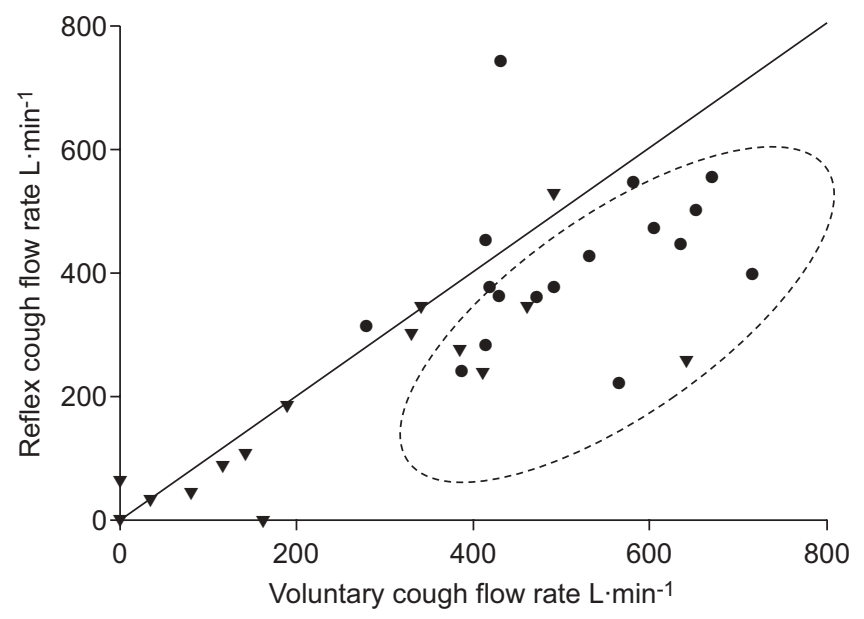

FIGURE 1. Reflex versus voluntary cough flow rate. $\mathbf{\nabla}$ : stroke patients;

- : control subjects; ---: individuals with lower reflex than voluntary cough flow rate; -: reference line, $y=x$.

reflex cough peak flow and voluntary cough peak flow, there are few individuals (highlighted in circle, both patients and controls) in whom reflex cough flow is lower than voluntary cough flow, but, contrary to Widdicombe and Addington's proposal, there was none in whom reflex cough flow rate was greater than voluntary cough.

\section{K. Ward}

Asthma, Allergy and Lung Biology, King's College London School of Medicine, London, UK.

Correspondence: K. Ward, Asthma, Allergy and Lung Biology, King's College London School of Medicine, The Chest Unit, 2nd Floor Cheyne Wing, King's College Hospital, Denmark Hill, London, UK. E-mail: katie.ward@kcl.ac.uk

Statement of Interest: None declared.

\section{REFERENCE}

1 Ward K, Seymour J, Steier J, et al. Acute ischaemic hemispheric stroke is associated with impairment of reflex in addition to voluntary cough. Eur Respir J 2010; 36: 1383-1390.

\section{Adequate use of pulmonary embolism clinical prediction rule in COPD patients}

\section{To the Editors:}

We read with interest the article by GUNEN et al. [1], entitled "Venous thromboemboli and exacerbations of COPD", recently published in the European Respiratory Journal (ERJ).

We congratulate the authors for their study, which is one piece of the puzzle of a persistent clinical dilemma: when and how do we have to search for a pulmonary embolism (PE) in chronic obstructive pulmonary disease (COPD) patients? One of their conclusions is that "the Wells criteria appear to be a significantly better tool [than the Geneva score] for exploring COPD patients for PE". This point raises some concerns, and readers of the ERJ should consider the following before applying this proposition.

This conclusion is mainly based on the distribution of PE among probability classes and comparison of receiver operating characteristic curves obtained with the Wells score and the Geneva score, both calculated in COPD patients hospitalised for an acute exacerbation. Contrary to the Wells score, which may be used in in- and outpatients, the Geneva score was initially derived and then validated only in outpatients. Therefore, its application in such selected hospitalised patients is hazardous and is not recommended [2]. This may explain the unexpected distribution of COPD patients among the different clinical classes, with only $10.6 \%$ of patients classified as low clinical probability when the expected proportion of patients in this subgroup is $\sim 50 \%$.
Notably, the Wells score is heavily influenced by the existence of an alternative diagnosis, and one can imagine that this point deeply decreases the external validity of the result of GUNEN et al. [1], who conducted a study focusing specifically on the prevalence of PE during COPD exacerbation. As COPD exacerbation may be easily integrated as an alternative diagnosis, it may reduce the reproducibility of these results among physicians in an emergency department, for example.

It is not clearly stated why the authors used quite outdated versions of both scores. Indeed, the Wells score [2, 3] has recently been submitted to revision and dichotomisation, with PE now considered as being "unlikely" or "likely". The same may be said for the Geneva score, which has been revised [4] a few years ago and simplified 2 yrs ago [5]. Blood gas analysis and chest radiography were excluded from the revised form. We can understand that it may be thought a waste of information for physicians aware of the issues, such as pneumologists, but the main objective of this revision was to provide an easier-to-use score, allowing clinical evaluation at bedside in the emergency department.

As clinical prediction rules (CPRs) tend to be underused [6], we think that revision and simplification of both rules matched the objective of rendering them easier to use. Therefore, new versions of these rules should be used. Notably, assessment of PE probability is the first step in the evaluation of PE suspicion and the use of a CPR is widely recommended [3]. According to 